\title{
THE PHARMACOTHERAPY TIME-TO-EFFECT OF OVERACTIVE BLADDER MEDICATIONS AND TREATMENT DURATION
}

\author{
MIKOŁAJ PRZYDACZ* and PIOTR CHŁOSTA
}

\author{
Department of Urology, Jagiellonian University Medical College, Krakow, Poland
}

\begin{abstract}
For Overactive Bladder $(\mathrm{OAB})$ treatment, pharmacotherapy is currently deemed as a second option. However, in the current publications related to $\mathrm{OAB}$, there is a significant research gap concerning the time-toeffect and duration of OAB pharmacotherapy. Such data is vital for effective and successful treatment of OAB, and hence, the study aimed to find information in this gap to assist healthcare providers in their daily activities in treating OAB. The study utilized the Web of Science databases, MEDLINE, Cochrane Library, and PubMed Central to find and collect the highest number of data. The literature review showed limited research concerning the time-to-effect and optimal treatment duration for OAB. However, based on the available data, it can be stated that the time-to-effect of OAB pharmacotherapy would be after seven days of the initial treatment. Besides, patients with $\mathrm{OAB}$ can be effectively treated for 6 to 12 months before the medications are stopped but patients need to be carefully educated about a risk of symptom reoccurrence and retreatment. Those who require further medications or have a higher risk of symptom reoccurrence may need lifetime pharmacotherapy or different treatment approaches. Further research is needed to focus on the time-to-effect during the first week of medication to accurately determine when first symptom reduction occurs, and carefully follow up for patients that show a positive response after the treatment has been ceased.
\end{abstract}

Keywords: OAB, pharmacotherapy, onset, time to effect, duration

Overactive Bladder $(\mathrm{OAB})$ refers to the condition where the patient consistently and frequently needs to urinate with or without urinary incontinence that has no correlations to urinary tract infection or other diseases (1). As such, the main reason patients seek treatment for $\mathrm{OAB}$ is the fact that their lifestyle becomes impacted significantly, especially concerning their mental health, sexuality, physical, social, and emotional wellbeing (2). Besides, OAB can touch a significant part of the patient's life, increasing its severity and need for treatment of the disease.

Over the years, antimuscarinic medications have been used as the primary treatment option for $\mathrm{OAB}$, and it mainly focuses on mitigating its symptoms. The use of antimuscarinic medications as a second-line treatment was supported by the Canadian Urological Association and European Association of Urology $(3,4)$. In addition to this, the beta-3-adrenoceptor agonist, mirabegron, has also been applied to treat $\mathrm{OAB}$ and is issued as a pharmacotherapy treatment for the disease. Nonetheless, healthcare providers need to conduct extensive tests, especially for proper diagnosis, possible contraindications, and adverse event profiles, to ensure that their diagnosis is correct and they select the best treatment for $\mathrm{OAB}$ per patient. Because a wide variety of comorbidities may produce or worsen $\mathrm{OAB}$ symptoms, diagnostic testing depends on the clinical scenario and often includes urine analysis, urine culture, voiding diary, specific questionnaires, bladder/renal ultrasound, cystoscopy, different imaging techniques (e.g., computed tomography, magnetic resonance imaging), or even urodynamic (pressureflow) study (3). The approach enhances the patientcentered care model while dealing with $\mathrm{OAB}$, but overall the medications applied for the disease are currently well tested, with documented adverse event profiles and contraindications.

However, a review of publications shows that there is limited research concerning the period OAB should be managed pharmacologically and the time to affect the medications utilized to treat the disease. Such data is crucial for the effective treatment of $\mathrm{OAB}$ and ensures that doctors and patients can improve their efficiency and performance in manag-

* Corresponding author: e-mail: mikolaj.przydacz@yahoo.com 
ing the condition. Also, it is known that $\mathrm{OAB}$ treatment compliance is an issue in routine clinical practice, thus, providing the above mentioned missing data would assist in improving the situation and boost patient conformity (5). Further, patient education is an essential principle in $\mathrm{OAB}$ and empowers patients to engage and participate in their treatment (3). As such, the research concentrates on providing data related to the period OAB should be managed pharmacologically, and the time to effect of the medication. The data will support physicians who care for $\mathrm{OAB}$ patients in their daily practice.

\section{MATERIALS AND METHODS}

The study utilized the Web of Science databases, MEDLINE, Cochrane Library, and PubMed Central to find and collect the highest number of data. The search was guided by reference checks and multiple keywords to ensure that the data was gathered objectively to minimize the risk of omission of essential studies. After that, the gather publications were reviewed manually, and the most related ones had their references checked to find other correlated publications that would be important for the study. Besides, the study chose to view currently available meta-analyses and systematic reviews that focused on pharmacotherapy of $\mathrm{OAB}$ to increase the data availability and validity of the research (5-14).

\section{RESULTS}

\section{The onset of medication efficacy}

The review of the publications found that data related to the time-to-effect of medications for $\mathrm{OAB}$ was minimal. The study identified that publications failed to identify and include clinical or statistical time data regarding the period when the OAB medications had made a significant impact on the disease symptoms. In the vast majority of trials, time-to-effect has not been considered as a primary or even secondary outcome. In addition, it was realized that the efficacy endpoints for most of the research protocols were assessed from just six to twelve weeks from the time they started treating the OAB.

\section{Antimuscarinics}

According to Khullar et al., the darifenacin made improvements to the patient's OAB symptoms at 6 to 8 days from the initial time treatment begun (15). Khullar et al. realized these findings in their pooled analysis of efficacy and safety for the darifenacin while analyzing the time-to-effect of the med- ication in a randomized to the drug or placebo investigation that had 1059 participants.

Similarly, a Canadian group sought to find the time-to-effect of medication for OAB (16). The Canadian group used fesoterodine $4 \mathrm{mg}$ compared to their placebo group and gathered 1013 participants with $\mathrm{OAB}$. The investigators found that fesoterodine $4 \mathrm{mg}$ made significant enhancement of the disease's symptoms concerning episodes of frequency, urgency, and urgency urinary incontinence, than the placebo approach after seven days of treatment.

Zinner et al. investigated the time-to-effect of $\mathrm{OAB}$ medications as done by the Canadian group, and Khullar et al. (17). Zinner et al., however, applied trospium chloride for the treatment approach for people suffering from OAB. Zinner et al. compared the drug to their placebo group and noted significant improvements. Zinner et al. discovered that in the daytime frequency, average volume per void, and average urge severity, the trospium chloride led to an initial seven-day improvement compared to the placebo approach. Also, the authors realized that the improvements to the endpoints were sustainable throughout the treatment period.

Sussman et al. analyzed the micturition frequency, urgency, and urge urinary incontinence endpoints (18). Sussman et al. utilized a tolterodine extended-release form. The authors used a post hoc analysis method and found that in all the assessed endpoints, the drug significantly improved frequency, urgency, and incontinence. Sussman et al. identified that the tolterodine's time-to-effect was five days and continued to increase into the next two days. Nonetheless, there some weaknesses in Sussman et al. approach as it failed to investigate efficacy before the fifth day, and there was no placebo control group.

Solifenacin was also analyzed in terms of the time-to-effect for OAB (19). The dosage was applied increasingly to identify when a significant impact where realized. The research also applied a randomized methodology with a placebo-controlled group and a double-blind effectiveness test. Solifenacin's time-to-effect was shown to be on the third day when $5 \mathrm{mg}$ of solifenacin was used per day.

Our study found that there was a research gap concerning the time-to-effect of oxybutynin and propiverine medications (5).

\section{Beta-3 adrenergic receptor agonist}

The time-to-effect of mirabegron was not effectively analyzed in any publications, and this needed to be addressed. The earliest beneficial 
impact of mirabegron was, however, identified to occur one week after the treatment begun in a second phase study (20). In addition, time to effect of the drug at $25 \mathrm{mg}$ and $50 \mathrm{mg}$ dosage was identified to significantly occur in four weeks compared to the placebo group. The medication showed significant improvement in frequency, mean volume per micturition, and incontinence.

Table 1 summarizes available data regarding the onset of treatment efficacy.

\section{Pharmacotherapy duration}

The study found that there was extensive research on clinical studies relating to OAB pharmacotherapy, yet there were few publications on treatment durations concerning the disease.

One-third of the OAB cases stated that the main reason for treatment for OAB was stopped because of the resolution of its bothersome symptoms (21-25). Besides, research also specified that other causes of OAB treatment termination were associated with adverse effects and failed prospective medical results. The study found that scholars and other stakeholders have not identified the best efficacy duration for $\mathrm{OAB}$ as the available data shows that in most randomized clinical trials it has been assessed to be between 2 weeks and twelve months, which is highly ambiguous (5-14). As such, it could be hypothesized that some research could underrate the drug effectiveness with short periods of drug administration (26-28), while the length of medication in other research could be longer than needed $(29,30)$. Added to these challenges is the fact that there is no standardized definition of refractory $\mathrm{OAB}$, which means that there are variations in the initial treatment periods for $\mathrm{OAB}$ with other medications or treatment modalities (31). Also, there is no consensus on when OAB pharmacotherapy should be ceased and the period when therapeutic efficacy is sustained after the medication has stopped.

\section{Antimuscarinics}

In another research, Hsiao et al. analyzed 164 participants issued with $4 \mathrm{mg}$ of tolterodine or $5 \mathrm{mg}$ of solifenacin prolonged capsule release medication for their OAB (32). Hsiao et al. observed the patient's progress for an average follow up of 1 month during a six-month space to determine the duration of needed treatment. The authors realized that for the anti-muscarinic administration, the medication period should be a minimum of 3 months. Choo et al. took a different approach and assessed the effects of drug cessation (33). Choo et al. evalu-
Table 1. Onset of medication efficacy for patients with overactive bladder.

\begin{tabular}{|c|c|}
\hline Medication & Time-to-effect \\
\hline Darifenacin & 6 days \\
\hline Fesoterodine & 7 days \\
\hline Mirabegron & 7 days \\
\hline Solifenacin & 3 days \\
\hline Tolterodine & 5 days \\
\hline Trospium & 7 days \\
\hline
\end{tabular}

The drugs are presented in alphabetic order.

ated 68 participants with $\mathrm{OAB}$ and focused on the efficacy of the treatment and the duration it took for the medication to initiate symptom reduction. Choo et al. measured changes in the participants that had been issued $20 \mathrm{mg}$ of propiverine hydrochloride administered daily and terminated after three-month medication. The authors found that after the four weeks' termination of treatment, there was a $35.3 \%$ retreatment rate recorded and attributed this to the increasing severity of the symptoms. In the retreatment group, older participants had higher scores for severe urgencies needed for the further treatment group. Under the urodynamic study, 23 participants showed detrusor overactivity and had higher rates of having the $\mathrm{OAB}$ symptoms reoccur after their treatment was stopped, but Choo et al. identified that the correlation was insignificant. As such, Choo et al. found that three months of $20 \mathrm{mg}$ of propiverine hydrochloride treatment was useful for $\mathrm{OAB}$, but once it was stopped, the symptoms had a higher probability of reoccurring after one month.

Khullar et al. supported Choo et al. findings on the cease period for $\mathrm{OAB}$ and its later effects on the patients (34). The British group analyzed 251 participants that received fesoterodine, four and eight $\mathrm{mg}$, and flexible dosage. The authors found that the symptoms of OAB reduced effectively after 12 weeks of the medication. However, the scholars also noted that the symptoms of $\mathrm{OAB}$ reoccurred after four weeks when its treatment was stopped. In addition, $61 \%$ of the patients experienced reduced health-related quality of life, increased micturition frequency, and severity of bladder-related problems. The British group then analyzed the escalation of treatment at the four-week period using a dosage of 4 to $8 \mathrm{mg}$ and found that it failed to reduce the rate at which the symptoms reoccurred. As such, it can be stated that the positive changes noted by antimuscarinic drug treatment on reduced $\mathrm{OAB}$ symptoms during a 12-week treatment period are not sustained after four weeks when the medications are stopped. 
In another study, tolterodine extended-release of $4 \mathrm{mg}$ daily dosage was used to treat OAB (21). The study utilized 108 participants that had the disease and developed a methodology that had three separate research groups that received the treatment at different intervals. The participants received their treatments for the OAB but ceased after a 1-, 3- or 6-months difference for each of the three groups. Later, they were reassessed after three months to determine the reoccurrence rate and significance of the OAB symptoms. The results showed that $65 \%$ of the participants needed further treatment, and $62 \%$ had a relapse of urgency, frequency, incontinence, and other symptoms. In addition, the study realized that the longer the duration of treatment, six months, there is still little improvement in the prevention of symptom relapse. The study, however, argued that with keen consultations with the patients about symptom relapse and retreatment, stopping medication was possible for those with improved symptoms status. The research also recommended practical patient-centered care approaches that analyze the patient's quality of life, especially those who are more likely to suffer from symptom relapse.

Kim et al. analyzed 371 participants that took antimuscarinic medication for a minimum of 12 weeks and had their symptoms subsided (35). The study stopped the treatment and viewed for symptom relapse for 1, 3, 6, and 12 months. One hundred seventy seven patients did not show recurrence at 6 months. Among this group, 41 participants agreed for further follow-up and just 4 patients of them $(9.7 \%)$ showed recurrence at 12 months; the other patients continued to be symptom-free up to the 12 months. Besides, the study showed that patients with concomitant urinary incontinence had a higher chance of reoccurrence.

Another study in Japan enrolled 73 participants using solifenacin $5 \mathrm{mg}$ administered daily for three years (25). The research found that $25 \%$ of them needed retreatment two times after ceasing the medication in ten months.

Yokoyama et al. compared patterns of treatment cessation of two different anticholinergics and showed that pharmacotherapy duration may fluctuate between various OAB medications (29). The authors prospectively randomized 109 OAB participants that had been issued $5 \mathrm{mg}$ of solifenacin once daily or $0.1 \mathrm{mg}$ imidafenacin twice daily with a twelve-month treatment plan. Twenty five percent of patients who canceled medicating with imidafenacin due to improvement required retreatment within 12 months compared to $53.8 \%$ of patients treated with solifenacin who also required drug reinitiation during the same time span.

A study in the USA, detailed that $34.6 \%$ of their patients, 103,250, needed retreatments after two years, $24.1 \%$ at one year (36). Also, $65.6 \%$ of the patients used their index anticholinergic agent, while the rest applied different approaches. The USA study utilized information from the IMS LifeLink Health Plan Claims Database.

\section{Beta-3 adrenergic receptor agonist}

Khrut et al. assessed the viability of ceasing mirabegron treatment after symptoms had decreased in OAB patients (37). Patients with an Urgency Bother-Visual Analog Scale score of $50 \%$ or less were counseled to stop mirabegron treatment at the 18 -month visit. The authors showed that 26.8 percent of the patients included stopped medicating with mirabegron without beginning it again at twenty-one months before the following check-up appointment.

\section{Physician perspective}

A Canadian group sought to investigate the practices utilized by urologists and gynecologists regarding the treatment of OAB (38). In the survey, the study found that $52 \%$ versus $45 \%$ of urologists, and gynecologists stated that its treatment should be time defined rather than lifelong. Also, urologists preferred limited treatment periods than gynecologists and were more likely to start medication with a higher dose. $41 \%$ of physicians preferred a sixmonth treatment period for $\mathrm{OAB}$, and $25 \%$ issued one-year medication time for the same disease.

\section{DISCUSSION}

The study conducted was the first to analyze the time-to-effect of medication and duration of treatment for OAB. As such, doctors and patients cannot develop practical treatment approaches without this information.

The study found a significant research gap for antimuscarinics and mirabegron time-to-effect for $\mathrm{OAB}$ through its clinical use had been extensively researched (5-14). Our research of the available limited data found that for these drugs, the time-toeffect was after seven days, but more investigation is needed to analyze this occurrence, especially in the first week of medication. Most of the studies that we analyzed were not designed to measure the time-toeffect.

Also, the optimal time for $\mathrm{OAB}$ treatment and avoidance of symptom reoccurrence has yet to be 
determined. However, the literature review section of the study explains that $\mathrm{OAB}$ treatment for 6 to 12 months may be effective. Nonetheles, in those with a higher risk of recurrence (particularly elderly, frail, with concomitant urinary incontinence, and detrusor overactivity presented in the urodynamic study) pharmacotherapy might be prolonged $(33,35)$. We can only speculate that it may go up to 18 months. Also, the patients should be enlightened to follow the medication procedure strictly throughout the period. After that, cessation of the treatment can be considered, but it needs to be individualistic towards the patients. Patients who are going to stop medications should be carefully counseled and educated about symptom relapse and retreatment. Those with a higher risk of reoccurrence should be informed that they have a higher risk of lifetime management.

The presented approach with personalized management might not fully comply with the standardization of treatment. However, treatment standardization in $\mathrm{OAB}$ is a significant issue and several shared pathways need to be considered. First, disparities have been noted between physicians' and patients' perceptions of the impact of a chronic disease such as OAB (39). Clinicians may underestimate the extent to which patients are affected by their symptoms in $25-37 \%$ of cases. Therefore, reports of medical outcomes after OAB treatment should include independent, validated questionnaires, self-administered by patients to avoid interviewer bias (40). Second, there is a significant impact of the placebo effect on OAB treatment outcomes (41). Current evidence from randomized trials shows that a positive placebo effect occurs in individuals treated for OAB. Finally, the reason being that $\mathrm{OAB}$ affects people uniquely, leading to diverse symptoms and other pathophysiologies.

The OAB should be identified as a multifaceted symptom syndrome that is caused by multiple pathophysiological features rather than simplifying it into idiopathic without factual support (42). The approach ensures that the treatment of $\mathrm{OAB}$ is an individualized lead distinction in the phenotypes of the disease. Also, this contributes to patient-centered care approaches that include treatment duration and time of primary efficacy.

The knowledge presented by the study would assist doctors to minimize their errors in developing medication approaches for their patients with OAB. As such, the administration of ineffective drugs and its contribution to symptom relapse may be avoided. The findings lead to one to hypothesize that information regarding the time-to-effect and duration of medicating OAB may lead to effective drug adher- ence by the patient. The study also acts as a reference point for future investigators that wish to focus on the time-to-effect and duration of OAB medication as available guidelines, including those by the Canadian Urological Association and European Association of Urology, have not described standards for these serious clinical concerns $(3,4)$. The current study recommends to future investigators to analyze the time-to-effect for the first week when treatment began to determine when symptom improvements occur at the earliest period. In addition, to assist physicians in their treatment plan for $\mathrm{OAB}$ patients, accurate evaluation with adequate follow-up when termination of care is needed, especially for people with positive medical results.

\section{CONCLUSION}

The time-to-effect of OAB medications should be expected after seven days of treatment to view significant changes to the patient's symptoms. Also, it can be recommended that $\mathrm{OAB}$ patients can receive medication for 6 to 12 months before canceling the treatment without expecting recurring symptoms. However, there must be specific and patient-centered counseling before the treatment is stopped. Patients that are at a higher risk of symptoms reoccurrence may need lifelong OAB pharmacotherapy or a shift in medications.

\section{Conflict of interest}

The authors declare no conflicts of interest.

\section{REFERENCES}

1. Abrams P., Cardozo L., Fall M., Griffiths D., Rosier P., et al.: Neurourol. Urodyn. 21, 167 2002).

2. Johnston K.M., Walker D.R., Lakzadeh P.: Adv. Ther. 36, 548 (2019).

3. Corcos J., Przydacz M., Campeau L., Gray G., Hickling D., et al.: Can. Urol. Assoc. J. 11, E142 (2017)

4. https://uroweb.org/guideline/urinary-incontinence/ (Cited: February 2020).

5. Buser N., Ivic S., Kessler T.M., Kessels A.G., Bachmann L.M.: Eur. Urol. 62, 1040 (2012).

6. Chapple C.R., Khullar V., Gabriel Z., Muston D., Bitoun C.E., Weinstein D.: Eur. Urol. 54, 543 (2008).

7. Usmani S.A., Reckenberg K., Johnson O., Stranges P.M., Teshome B.F., et al.: Drugs Aging 36, 639 (2019). 
8. Yeowell G., Smith P., Nazir J., Hakimi Z., Siddiqui E., Fatoye F.: BMJ Open 8, e021889 (2018).

9. Kelleher C., Hakimi Z., Zur R., Siddiqui E., Maman K., et al.: Eur. Urol. 74, 324 (2018).

10. Kistler K.D., Xu Y., Zou K.H., Ntanios F., Chapman D.S., Luo X.: Neurourol. Urodyn. 37, 54 (2018).

11. Reynolds W.S., McPheeters M., Blume J., Surawicz T., Worley K., et al.: Obstet. Gynecol. 125, 1423 (2015).

12. Maman K., Aballea S., Nazir J., Desroziers K., Neine M.E., et al.: Eur. Urol. 65, 755 (2014).

13. Madhuvrata P., Cody J.D., Ellis G., Herbison G.P., Hay-Smith E.J.: Cochrane Database Syst. Rev. 1, Cd005429 (2012).

14. Sexton C.C., Notte S.M., Maroulis C., Dmochowski R.R., Cardozo L., et al.: Int. J. Clin. Pract. 65, 567 (2011).

15. Khullar V., Foote J., Seifu Y., Egermark M.: Int. Urogynecol. J. 22, 1573 (2011).

16. Corcos J., Angulo J.C., Garely A.D., Carlsson M., Gong J., Guan Z.: Curr. Med. Res. Opin. 27, 1059 (2011).

17. Zinner N., Gittelman M., Harris R., Susset J., Kanelos A., Auerbach S.: J. Urol. 171, 2311 (2004)

18. Sussman D.O., Kraus S.R., Carlsson M., Guan Z.: Curr. Med. Res. Opin. 23, 777 (2007).

19. Cardozo L., Hessdorfer E., Milani R., Arano P., Dewilde L., et al. BJU Int. 102, 1120 (2008).

20. Chapple C.R., Nitti V.W., Khullar V., Wyndaele J.J., Herschorn S., et al.: World J. Urol. 32, 1565 (2014).

21. Lee Y.S., Choo M.S., Lee J.Y., Oh S.J., Lee K.S.: Int. J. Clin. Pract. 65, 997 (2011).

22. Sung H.H., Han D.H., Kim T.H., Lee Y.S., Lee H.N., et al.: Int. J. Clin. Pract. 69, 1309 (2015).

23. Krhut J., Gartner M., Petzel M., Sykora R., Nemec D., et al.: Scand. J. Urol. 48, 79 (2014).

24. Shim E.J., Yoo E.H., Kim Y.M., Kim D.: Obstet. Gynecol. Sci. 58, 507 (2015).
25. Tanaka Y., Tanuma Y., Masumori N.: Int. J. Urol. 23, 866 (2016).

26. Herschorn S., Stothers L., Carlson K., Egerdie B., Gajewski J.B., et al.: J. Urol. 183, 1892 (2010).

27. Altan-Yaycioglu R., Yaycioglu O., Aydin Akova Y., Guvel S., Ozkardes H.: Br. J. Clin. Pharmacol. 59, 588 (2005).

28. Jacquetin B., Wyndaele J.: Eur. J. Obstet. Gynecol. Reprod. Biol. 98, 97 (2001).

29. Yokoyama T., Koide T., Hara R., Fukumoto K., Miyaji Y., Nagai A.: Urol. Int. 90, 161 (2013).

30. Halaska M., Ralph G., Wiedemann A., Primus G., Ballering-Bruhl B., et al.: World J. Urol. 20, 392 (2003).

31. Nitti V.W., Kopp Z., Lin A.T., Moore K.H., Oefelein M., Mills I.W.: Neurourol. Urodyn. 29, 652 (2010).

32. Hsiao S.M., Liao C.H., Lin H.H., Kuo H.C.: Int. Neurourol. J. 19, 171 (2015).

33. Choo M.S., Song C., Kim J.H., Choi J.B., Lee J.Y., et al.: J. Urol. 174, 201 (2005).

34. Khullar V., Cardozo L., Kelleher C.J., Hall T., Ryan J., et al.: BJU Int. 112, 820 (2013).

35. Kim A., Lee K.S., Kim T.B., Kim H.J., Yoo E.S., et al.: Investig. Clin. Urol. 58, 42 (2017).

36. Chancellor M.B., Migliaccio-Walle K., Bramley T.J., Chaudhari S.L., Corbell C., Globe D.: Clin. Ther. 35, 1744 (2013).

37. Krhut J., Martan A., Zachoval R., Hanus T., Horcicka L., et al.: Int. Urol. Nephrol. 50, 433 (2018).

38. Przydacz M., Campeau L., Walter J.E., Corcos J.: Can. Urol. Assoc. J. 12, E378 (2018)

39. Rodriguez L.V., Blander D.S., Dorey F., Raz S., Zimmern P.: Urology 62, 49 (2003).

40. Przydacz M., Golabek T., Chlosta P.: Adv. Clin. Exp. Med. 28, 555 (2019).

41. Mangera A., Chapple C.R., Kopp Z.S., Plested M.: Nat. Rev. Urol. 8, 495 (2011).

42. Peyronnet B., Mironska E., Chapple C., Cardozo L., Oelke M., et al.: Eur. Urol. 75, 988 (2019).

(C) 2020 by Polish Pharmaceutical Society. This is an open-access article under the CC BY NC license (c) (i) (9) (http://creativecommons.org/licenses/by-nc/4.0/). 